\title{
Spurious Rotor Detection During Atrial Fibrillation: Phase Singularities in Fact Reflect Blurred Conduction Block
}

\author{
Stef Zeemering ${ }^{1}$, Piotr Podziemski ${ }^{1}$, Pawel Kuklik ${ }^{2}$, Arne van Hunnik ${ }^{1}$, Bart Maesen ${ }^{1}$, Ulrich \\ Schotten ${ }^{1}$ \\ ${ }^{1}$ Department of Physiology, Cardiovascular Research Institute Maastricht, Maastricht University, \\ Maastricht, The Netherlands \\ ${ }^{2}$ Department of Cardiology, University Hospital Eppendorf, Hamburg, Germany
}

\begin{abstract}
Rotors during atrial fibrillation $(A F)$ have been implicated as potential targets for AF ablation. Analysis in the phase domain to detect phase singularities (PS) may however blur the underlying wave front activation pattern, and obscure lines of conduction block. We investigated the coincidence of PS trajectories and lines of conduction block to assess the disruptive effect of phase reconstruction methods on the underlying propagation pattern and rotor detection accuracy. High-density unipolar electrograms were recorded in 20 patients with $A F$ during cardiac surgery, using an electrode array $(25 \times 25 \mathrm{~mm})$ placed on the left posterior and right atrial wall. Activation maps were constructed using probabilistic local deflection detection; phase maps were reconstructed using sinusoidal recomposition or frequency-based filtering, followed by phase computation using Hilbert transform or time-delay embedding. Conduction block was defined as local conduction velocity $<10 \mathrm{~cm} / \mathrm{s}$ between adjacent electrode activations. In 38 recordings (10 seconds), 138 sustained PS trajectories were detected (lifespan $>1$ atrial cycle), using sinusoidal recomposition and Hilbert transform, out of which 130 (94\%) were consistently within 1 electrode distance $(1.5 \mathrm{~mm})$ of a line of conduction block. In contrast, concurrent points in the mapping array were consistently further away from a line of block than the detected $P S$ points (median difference $+2.1 \mathrm{~mm}, p \ll 0.001$ ). Other approaches to reconstruct phase maps yielded comparable results. The far majority of rotors detected in phase maps are co-located with a line of block in activation maps, not with a rotating wave front, showing that rotor detection by phase singularity tracking is a non-specific method in the presence of conduction block. Our results may explain why ablation at the core of a rotor can sometimes be successful.
\end{abstract}

\section{Introduction}

Atrial fibrillation (AF) is the most common sustained cardiac arrhythmia. It is associated with substantial increase in stroke risk and reduced quality of life.[1] Catheter ablation has been developed to identify and eliminate sites or regions that trigger or sustain AF. Rotors, defined as sustained phase singularity points (PS), have been implicated as candidate drivers of AF, and consequently as potential targets for AF ablation. Several studies have reported localized rotors that putatively perpetuate AF in patients, which were detected by phase mapping of AF using endocardial mapping catheters[2] or electrocardiographic imaging.[3] Analysis in the phase domain may however blur the underlying wave front activation pattern, and critically - lines of conduction block. As a consequence, a location where separate wave fronts propagate alongside a line of conduction block in various directions could be interpreted as a phase singularity in a corresponding phase map. To assess the disruptive effect of electrogram filtering and phase transform methods on the interpretation and characterization of the underlying propagation pattern and rotor detection accuracy, we investigated the coincidence of PS trajectories in phase maps and lines of conduction block in activation time maps.

\section{Methods}

\subsection{Materials and electrogram processing}

Electrograms were recorded by means of high-density contact mapping of AF using a $16 \times 16$ grid of electrodes (1.5 mm electrode distance) in 20 patients during openchest cardiac surgery. Epicardial unipolar electrograms were acquired from both the right atrial free wall and posterior left atrium at a sampling frequency of $1 \mathrm{kHz}$ for 10 seconds. Patients were in paroxysmal AF (PAF, $n=11)$ or persistent $\mathrm{AF}$ (persAF, $\mathrm{n}=9$ ) at the time of recording. Elec- 
trograms were not filtered before analysis, but ventricular contributions to the atrial electrograms, if present, were removed using a single beat cancellation method, by removing the first principal component as computed by principal component analysis on single ventricular beats (detected in a reference ECG), under the assumption that the first principal component represented the ventricular contribution.

\subsection{Activation time annotation and con- duction block detection}

Local atrial activation times were annotated by an algorithm that uses template matching and maximum activation interval likelihood assignment.[4] Candidate deflections were marked by template matching. Based on the largest and steepest candidate deflections, an estimate of the underlying probability density function of the AF cycle length was determined. Deflections were then assigned either as true local activations or as remote deflections to maximize the likelihood of the local activation intervals given the estimated AF cycle length distribution. Local conduction block was defined as a difference in local activation time between activations at two adjacent electrodes that resulted in a conduction velocity slower than a threshold conduction velocity $\mathrm{CV}_{\text {block }}=10 \mathrm{~cm} / \mathrm{s}$. Conduction block duration was defined as the interval from the incidence of conduction block to the next occurrence of normal conduction ( $\mathrm{CV} \geq \mathrm{CV}_{\text {block }}$ ). Furthermore, local conduction block within a wavefront was ignored, meaning that conduction block was only present between adjacent electrode activations if no alternative path with normal conduction could be found. Constructed in this way, isolated conduction block locations form connected lines of conduction block that separate the unconnected wavefronts that propagate over the mapping area.

\subsection{Phase reconstruction and singularity detection}

Electrogram phase was estimated by any combination of two filtering methods, followed by two phase reconstruction methods. The two filtering methods were sinusoidal recomposition[5] and a recently described frequency domain filter that emphasises local deflection energy.[6] The two phase reconstruction methods were the Hilbert transform and time-delay embedding, the latter with a time delay of $25 \%$ of the AF cycle length. Locations containing a PS were detected using estimated phase signals, following the approach described by Kuklik et al.[7], where a PS is only detected if a sudden phase transition $>\pi$ occurs in two paths encircling a point, in this case formed by electrodes at $1.5 \mathrm{~mm}$ and $3 \mathrm{~mm}$ distance. Furthermore, to limit the analysis to sustained rotational activity, a PS was only analyzed if the lifespan of its trajectory exceeded the local AF cycle length.

\subsection{Distance between a phase singularity and a line of conduction block}

For every detected PS trajectory, the distance to nearest co-occurring line of conduction block was determined for each time instant during the trajectory lifespan. To quantify the probability that the resulting distances occur by chance, the average distance of a PS to a line of conduction block was then compared to the average distance of every location where no PS was detected (non-PS locations) to the nearest line of conduction block during the lifespan of the PS trajectory. The paired differences in the average distance to a line of conduction block between PS and non-PS locations were tested using the Wilcoxon signedrank test.

\section{Results}

A total of 38 recordings were analyzed. An example of conduction block detection in activation time maps and PS detection in phase maps is shown in Figure 1. To investigate how electrogram processing and filtering influence phase singularity detection at the line of conduction block, we compared sinusoidal recomposition with frequencybased filtering. As a substitute of Hilbert transform for phase signal construction, we used time-delay embedding. We applied all four combinations of electrogram processing to reconstruct electrogram phase. The average distance of a detected PS to the nearest conduction block was calculated with all the four possible combinations of methods. For all tested method combinations, on average the PS locations were found to be significantly closer to a line of conduction block than non-PS locations (see Table 1), even though the number of detected PS varied between methods. Moreover, using sinusoidal recomposition and Hilbert transform, 130 out of 138 detected PS trajectories (94\%) were consistently within 1 electrode distance $(1.5 \mathrm{~mm})$ of a line of conduction block. By contrast, only $15 \%$ of all non-PS locations were on average within 1 electrode distance of the nearest line of conduction block ( $\mathrm{p} \ll 0.001$, Fisher's exact test).

\section{Conclusions}

These results indicate that detecting rotors using phase analysis on complex electrograms recorded during AF can lead to spurious results. Phase singularities often coincide with a conduction pattern of two or more wavefronts that propagate alongside a line of conduction block. The analysis of conduction patterns always requires various degrees of electrogram preprocessing, such as filtering. The 


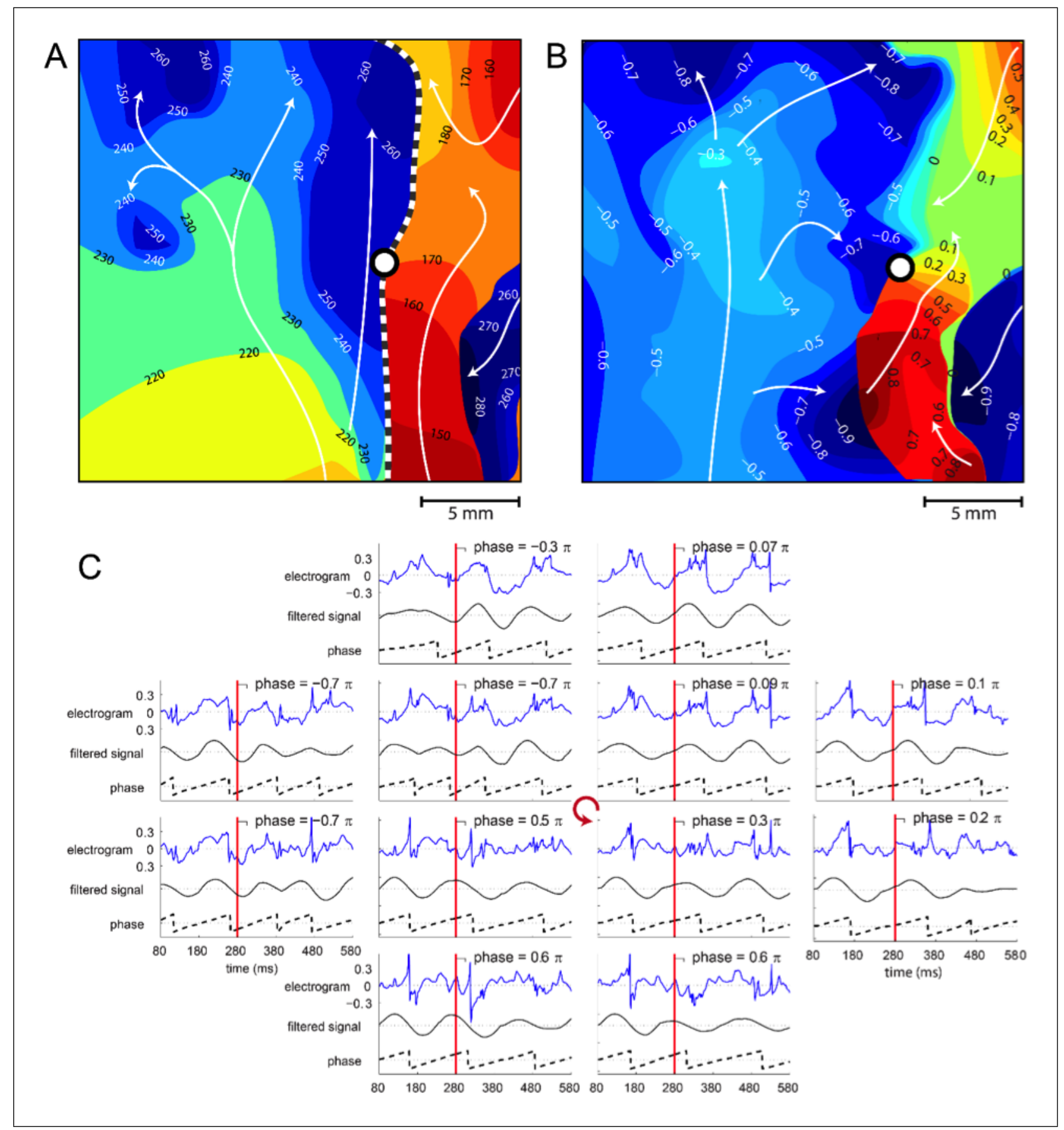

Figure 1. Example of conduction block detection (dotted line) in an activation time wave front map (A) and phase singularity detection (white dot) in a corresponding electrogram phase map (B). In (C) the electrograms are shown (blue) together with the filtered signals after sinusoidal recomposition (black) and phase signals after the Hilbert transform (black, dotted) for the electrodes around the detected PS. The vertical red lines in (C) indicate the latest activation time shown in (A). It can be appreciated that the reconstructed phase map in (B) alters the interpretation of the of the propagation pattern as observed in the wave front propagation map in (A), and that the detected PS is located on a line of conduction block. 
Table 1. Distance of PS and non-PS locations to a site of conduction block using different phase reconstruction methods

\begin{tabular}{|c|c|c|c|c|}
\hline method combination & number of PS & $d_{\mathrm{PS}}[\mathrm{P} 5-\mathrm{P} 95]$ & $d_{\text {non-PS [P5-P95] }}$ & paired difference [95\% CI] \\
\hline $\mathrm{SR}-\mathrm{HT}$ & 138 & $1.1 \mathrm{~mm}[0.0-5.9 \mathrm{~mm}]$ & $3.8 \mathrm{~mm}[1.4-8.2 \mathrm{~mm}]$ & $2.1 \mathrm{~mm} *[1.6-2.4 \mathrm{~mm}]$ \\
\hline $\mathrm{FF}-\mathrm{HT}$ & 107 & $1.4 \mathrm{~mm}[0.1-5.9 \mathrm{~mm}]$ & $3.2 \mathrm{~mm}[1.3-7.2 \mathrm{~mm}]$ & $1.3 \mathrm{~mm}^{*}[1.1-2.0 \mathrm{~mm}]$ \\
\hline SR - TE & 80 & $1.2 \mathrm{~mm}[0.1-4.6 \mathrm{~mm}]$ & $3.8 \mathrm{~mm}[1.4-7.4 \mathrm{~mm}]$ & $2.2 \mathrm{~mm}^{*}[1.8-2.6 \mathrm{~mm}]$ \\
\hline FF - TE & 45 & $2.1 \mathrm{~mm}[0.1-5.4 \mathrm{~mm}]$ & $4.0 \mathrm{~mm}[1.5-7.9 \mathrm{~mm}]$ & $2.0 \mathrm{~mm}^{*}[1.3-2.9 \mathrm{~mm}]$ \\
\hline
\end{tabular}

PS: Phase Singularity; SR: Sinusoidal Recomposition; HT: Hilbert Transform; FF: Frequency Filter; TE: Time-delay Embedding; $d_{\mathrm{PS}}, d_{\text {non-PS}}$ : median average distance of (non)-PS locations to a site of conduction block; CI: Confidence Interval; $* \mathrm{p} \ll 0.001$ using a Wilcoxon signed-rank test.

discrepancy between the interpretation of conduction patterns produced by phase map analysis and direct activation time map in this study shows how much filtering affects our perception of the conduction pattern during AF. The apparent co-location of PS locations and regions with conduction block may explain why ablation at the core of a rotor can sometimes be successful. PS locations may exhibit a higher probability of conduction block, and do not necessarily reflect areas where true rotors may occur. These areas of a higher probability of conduction block could contribute to wave generation and increase AF complexity. Elimination of a such a pro-arrhythmic site by ablation may reduce the complexity of the AF propagation pattern and eventually lead to termination of fibrillatory conduction.

\section{Acknowledgements}

This work was supported by the Netherlands Heart Foundation (CVON2014-09, RACE V: Reappraisal of Atrial Fibrillation: Interaction between hyperCoagulability, Electrical remodeling, and Vascular Destabilization in the Progression of AF), and the European Union (European Network for Translational Research in Atrial Fibrillation, FP7 collaborative project, No. 261057; CATCH ME: Characterizing Atrial fibrillation by Translating its Causes into Health Modifiers in the Elderly, No. 633196; the ITN Network AFibTrainNet, No. 675351; the ITN Network RADOX: RADical reduction of OXidative stress in cardiovascular diseases, No. PITN-GA-2012-316738; and the ERACoSysMED H2020 ERA-NET Cofund project Systems medicine for diagnosis and stratification of atrial fibrillation)

\section{References}

[1] Andrade J, Khairy P, Dobrev D, Nattel S. The clinical profile and pathophysiology of atrial fibrillation: relationships among clinical features, epidemiology, and mechanisms. Circulation Research April 2014;114(9):1453-1468.

[2] Narayan SM, Krummen DE, Shivkumar K, Clopton P, Rappel WJ, Miller JM. Treatment of atrial fibrillation by the ablation of localized sources: CONFIRM (Conventional Ab- lation for Atrial Fibrillation With or Without Focal Impulse and Rotor Modulation) trial. Journal of the American College of Cardiology August 2012;60(7):628-636.

[3] Haïssaguerre M, Hocini M, Denis A, Shah AJ, Komatsu Y, Yamashita S, Daly M, Amraoui S, Zellerhoff S, Picat MQ, Quotb A, Jesel L, Lim H, Ploux S, Bordachar P, Attuel G, Meillet V, Ritter P, Derval N, Sacher F, Bernus O, Cochet H, Jaiis P, Dubois R. Driver domains in persistent atrial fibrillation. Circulation August 2014;130(7):530-538.

[4] Zeemering S, Maesen B, Nijs J, Lau DH, Granier M, Verheule S, Schotten U. Automated quantification of atrial fibrillation complexity by probabilistic electrogram analysis and fibrillation wave reconstruction. Conference proceedings Annual International Conference of the IEEE Engineering in Medicine and Biology Society IEEE Engineering in Medicine and Biology Society Conference 2012;2012:63576360.

[5] Kuklik P, Zeemering S, Maesen B, Maessen J, Crijns HJ, Verheule S, Ganesan AN, Schotten U. Reconstruction of instantaneous phase of unipolar atrial contact electrogram using a concept of sinusoidal recomposition and Hilbert transform. IEEE Transactions on Biomedical Engineering January 2015;62(1):296-302.

[6] Roney CH, Cantwell CD, Qureshi NA, Chowdhury RA, Dupont E, Lim PB, Vigmond EJ, Tweedy JH, Ng FS, Peters NS. Rotor Tracking Using Phase of Electrograms Recorded During Atrial Fibrillation. Annals of biomedical engineering December 2016;.

[7] Kuklik P, Zeemering S, van Hunnik A, Maesen B, Pison L, Lau DH, Maessen J, Podziemski P, Meyer C, Schaffer B, Crijns H, Willems S, Schotten U. Identification of Rotors during Human Atrial Fibrillation Using Contact Mapping and Phase Singularity Detection: Technical Considerations. IEEE Transactions on Biomedical Engineering February 2017;64(2):310-318.

Address for correspondence:

Stef Zeemering

P.O. Box 616, 6200 MD, Maastricht, The Netherlands

s.zeemering@maastrichtuniversity.nl 